\title{
SOME ASPECTS OF THE PROBLEM OF MATHEMATICAL RIGOR ${ }^{1}$
}

\author{
HASKELL B. CURRY
}

1. Introduction. The cue for the title to this address is taken from that of one by Pierpont before the Nashville meeting of this Society several years ago. ${ }^{2}$ This is typical of a number of expository treatments of this topic which have been presented to the mathematical public in recent years. ${ }^{3}$ In the present paper I shall discuss the same theme in a somewhat different manner. Relying upon these expository addresses for the historical background, I propose to treat certain aspects of the subject which have been rather neglected in them. The discussion is frankly from a single point of view, which is a species of formalism. I shall try, in the first place, to explain the fundamental concepts of formalism, and, in the second place, to add some new suggestions and criticisms in matters of detail. ${ }^{4}$

The problem of mathematical rigor is that of giving an objective definition of a rigorous proof. If you will examine your ideas on this subject I think you will agree that there is something vague and subjective about them. This does not mean, of course, that they are unsatisfactory for the needs of working mathematicians. In daily life, when we say that a piece of cloth is a yard wide, we really mean that its width is a certain legally defined fraction of the distance between two scratches on a metal bar located in Paris; nevertheless we do not rush to Paris when we wish to verify that a piece of cloth has this property. Secondary standards of varying degrees of accuracy suffice for the needs of daily life and of science; but neither science nor business would be possible without exact primary standards. Even so we need a primary standard of rigor in mathematics. The definition of such a standard, and the elaboration of practical secondary standards

\footnotetext{
${ }^{1}$ An address delivered before the meeting of the Society in New York City on October 26, 1940, by invitation of the Program Committee.

${ }^{2} \mathrm{~J}$. Pierpont, Mathematical rigor, past and present, this Bulletin, vol. 34 (1928), pp. 23-53.

${ }^{3}$ See for example A. Dresden, Some philosophical aspects of mathematics, this Bulletin, vol. 34 (1928), pp. 438-452; G. H. Hardy, Mathematical proof, Mind, vol. 38 (1929), pp. 1-25; E. R. Hedrick, Tendencies in the logic of mathematics, Science, vol. 77 (1933), pp. 335-343.

${ }^{4}$ For views related to those here presented, see my paper Remarks on the definition and nature of mathematics, Journal of Unified Science, vol. 9, pp. 164-169. This is an abstract of an address delivered before the Fifth International Congress of Unified Science at Cambridge, Massachusetts, September 5, 1939.
} 
in relation to it, constitutes the outstanding problem of the foundations of mathematics.

This problem is evidently tantamount to finding exact criteria for the truth of a mathematical proposition. For we should regard a rigorous proof as a process of making manifest the truth of its conclusion; and the problem of rigor is inseparable from that of truth.

2. Critique of the non-formal theories of mathematics. There are three main types of view as to the nature of mathematical truth; these I shall call realism, idealism, and formalism. The first of these is the view that mathematical propositions concern the real world (in the sense of our physical environment); the second holds that mathematics deals with mental objects of some sort. These two views have in common the notion that mathematical propositions have an essential subject-matter or content, with respect to which truth and rigor are definable; they are what the Germans would call "inhaltlich." Since there is no satisfactory translation of this word into English, ${ }^{5}$ I propose that we call them contensive, where 'contensive' is derived from 'content' in the same way as 'intensive' from 'intent.' The formalist view will form the main subject of the present paper; but before we take it up it will be appropriate to consider a few remarks about the others.

The realist view evidently does not need to be taken seriously at the present time. Of course it is the original view-the mathematics of primitive peoples is essentially empirical - and it is tenable for simple arithmetic propositions such as a $2+2=4$. But that the infinitistic conceptions of modern mathematics have no counterpart in the external environment is a point which needs no elaboration.

The idealist view has many varieties according to the nature of the mental objects on which it is based. On the one hand there is the view, called by Bernays ${ }^{6}$ Platonism, which ascribes an independent existence to all the infinitistic conceptions of mathematics; at the other extreme there is intuitionism, which denies this existence, but bases everything on an a priori intuition. Whatever the nature of these mental objects, all forms of idealism are subject to the same fundamental criticism, namely, that the criterion of truth is vague at best, and the existence of the mental objects is a metaphysical hypoth-

\footnotetext{
5 Translations sometimes used are 'material' and 'intuitive'; but these have undesirable connotations.

${ }^{6} \mathrm{P}$. Bernays, Sur le platonisme dans les mathématiques, L'Enseignement Mathématique, vol. 34 (1935-1936), pp. 52-69. There are naturally many different varieties of Platonism and also, for that matter, of intuitionism.
} 
esis from which mathematics should be free, if it is to be an objective science.

That this criticism applies to Platonism has been shown by its intuitionist critics, and is now fairly generally admitted. But it also applies to more moderate forms of idealism, and even to intuitionism itself. For the vagueness of the fundamental "oerintuitie der wiskunde" has been noticed by several persons; and Heyting himself, in his Ergebnisse report of 1934, explicitly denies the possibility of describing it exactly. ${ }^{7}$ On the other hand it is clear from the intuitionists' writings that they conceive their fundamental intuition to be (1) essentially a thinking activity, ${ }^{8}$ (2) a priori, ${ }^{9}$ (3) independent of language, ${ }^{10}$ and (4) objective, in that it is the same in all thinking beings. ${ }^{11}$ The existence of an intuition having these properties-or even the first three of them-is an outright assumption. It is doubtful, indeed, if there is any a priori knowledge; and it has been rationally maintained that thinking of any kind is impossible without language. Furthermore the fourth property, although it is absolutely vital if intuitionism is to give any account of mathematical truth at all, seems patently incompatible with the other three. In short, the intuitionist definition of mathematics has meaning only for one who postulates an a priori intuition which is both objective and pre-linguistic. Although such a postulate is agreeable to certain types of philosophy, yet it is an assumption for all that; and one which, from other points of view, is highly dubious and metaphysical.

The intuitionist school has made contributions of great value to the foundations of mathematics. Not only have they cleared away much of the rubbish of old-fashioned Platonism, but they have helped to sharpen the ideas of the formalists. We shall see later that most of the constructive suggestions of the intuitionists, when cleared of their metaphysics, find their justification in modern formalism. But

${ }^{7}$ A. Heyting, Mathematische Grundlagenforschung, Intuitionismus, Beweistheorie, Ergebnisse der Mathematik und ihre Grenzgebiete, vol. 3, no. 4, Berlin, Springer, 1934. See for example, page 12 where he writes "Überdies ist es an sich widersinnig, die Möglichkeiten des Denkens in das Mieder bestimmter zuvor angebener Konstruktionsprinzipien zwängen zu wollen. Man muss sich also darauf beschränken, durch mehr oder weniger vage Umschreibungen in dem Hörer die mathematische Geisteshaltung hervorzurufen."

8 "Eine konstruktive Tätigkeit unseres Verstandes" (Heyting, loc. cit., p. 2).

9 "Die mathematischen Gegenstände werden von dem denkenden Geist unmittelbar erfasst; die mathematische Erkenntnis ist daher von der Erfahrung unabhängig." (Heyting, loc. cit., p. 3.)

${ }^{10}$ Cf. Heyting, loc. cit., p. 13.

${ }^{11}$ A fifth characteristic, its relation to time, is irrelevant for us. 
the fundamental basis of their theory is a metaphysical doctrine.

Before leaving the subject of contensive definitions it will be necessary to consider the term logicism (or logisticism). It is sometimes maintained that there are three principal views as to the foundations of mathematics: intuitionism, formalism, and logicism, where the last is the view that mathematics is reducible to logic. If we had a satisfactory definition of logical truth and of logical rigor, logicism would solve our problem very nicely. But the paradoxes have shown that this is not the case; and in fact logic is as much in need of definition as mathematics. Hence to say that mathematics is logic is merely to replace one undefined term by another. Practically all competent writers realize this; and those who maintain that mathematics is logic generally couple this contention with some explanation of what logic is. But when we take these definitions of logic into account, the resulting definitions of mathematics are not a unified view according to the classification here adopted. Ramsey was a Platonist; Frege was essentially a formalist, and most of the modern writers who are popularly classified as logicists are definitely so. 'Logicism,' then, is not the name of a distinct view in regard to the foundations of mathematics.

From these considerations we see that a satisfactory contensive definition of mathematical truth and rigor has not been made; and indeed there is reasonable doubt as to whether such a definition is possible. Although such conceptions are current among mathematicians, they are useful only as secondary standards.

3. The notion of formal system. This brings us to formalism. According to this view the emphasis is not on the content of mathematical propositions, but on the method of derivation. We have an exact definition of a rigorous proof, and identify truth with the existence of such a proof.

The precise analysis of this rather vague definition requires preliminary consideration of some basic concepts. The first of these concepts is the notion of formal system. ${ }^{12}$ This is a refinement of the naive notion of a postulate system, and it will be convenient to introduce the new notion by taking an example of the latter notion and formalizing it. The example selected is Dickson's postulate set for group theory, ${ }^{13}$ as follows:

12 This statement is meant in the sense that the notion of formal system is taken as basic in the present paper, not that it is characteristic of formalism as such. Cf. below, $\$ 5$.

${ }^{13}$ L. E. Dickson, Definition of a group and a field by independent postulates, Transactions of this Society, vol. 6 (1905), pp. 198-204. 


\section{DICKSON'S POSTULATES FOR GROUPS}

A. Primitive ideas. $G$, o.

B. Postulates.

1. If $a$ and $b$ are elements of $G$, then $a \circ b$ is a uniquely determined element of $G$.

2. If $a, b, c$ are any elements of $G$, then

$$
a \circ(b \circ c)=(a \circ b) \circ c .
$$

3. There exists an element $e$ of $G$, such that for any element $a$ of $G$

$$
a \circ e=a \text {. }
$$

4. For each element $a$ of $G$ there exists an element $a^{\prime}$ such that

$$
a \circ a^{\prime}=e \text {. }
$$

This set of postulates determines a set of theorems which are derived from them by the processes of logical deduction. But, as we have seen in the discussion of logicism, this is not an objective characterization. It is necessary to specify in detail the way in which theorems are to be derived.

A formal system may be naively described as what such a postulate system becomes when we put in these refinements. More precisely, such a system is defined by a set of conventions, which I shall call its primitive frame. These conventions are of three kinds, as follows : first, those which specify the objects of the system, which I shall call its terms - in the case of group theory these are the elements of the group; second, those determining a set of propositions, which I shall call the elementary propositions, concerning these terms-in the case of group theory these are the equations; and third, those which specify which of the elementary propositions are true, that is, are theorems. ${ }^{14}$ These specifications have the form of recursive definitions. Thus the termspecifications give a list of primitive terms, or tokens, together with operations and rules of term formation which describe how further terms are to be constructed from the tokens. The specifications for elementary propositions consist of a list of predicates (properties, relations, and so on) together with rules of proposition formation for constructing elementary propositions by means of them. Finally, the specifications for theorems consist of a set of elementary propositions, called axioms, which are stated outright to be true; together with rules of deduction showing how further theorems are to be derived.

${ }^{14}$ Throughout this paper I shall use 'theorem' in the sense of 'true proposition.' Thus an axiom is a theorem by definition. 
As in recursive definitions generally a property of closure is understood, namely, that the specifications give all the entities recursively -for example, all the terms are obtained by the processes described in the term specifications, and so on.

For a system $G$, related to group theory, the primitive frame is as follows :

I. TERMs.

\section{System G}

A. Primitive terms, or tokens. $e, a_{1}, \cdots, a_{n}$.

B. Operations. o (binary), ' (unary).

C. Rules of term formation.

1. If $A$ and $B$ are terms, $A \circ B$ is a term.

2. If $A$ is a term, $A^{\prime}$ is a term.

II. Elementary propositions.

A. Predicates. = (binary).

B. Rule. If $A$ and $B$ are terms, then $A=B$ is an elementary proposition. ${ }^{15}$

III. TheOREMS.

A. Axioms. If $A, B, C$ are terms, then-

1. $A \circ(B \circ C)=(A \circ B) \circ C$,

2. $A \circ e=A$,

3. $A \circ A^{\prime}=e$.

B. Rules of deduction. For any terms $A, B, C-$

1. If $A=B$, then $B=A$.

2. If $A=B$ and $B=C$, then $A=C$.

3. If $A=B$, then $C \circ A=C \circ B$.

4. If $A=B$, then $A \circ C=B \circ C$.

5. If $A=B$, then $A^{\prime}=B^{\prime}$.

The following remarks are intended to clear up certain points in regard to the primitive frame. (What a formal system essentially is will concern us later; for the present we are interested only in the specifications by which a formal system is defined.)

First, as to the symbols used. In the above statement of the primitive frame the symbols ' $e$,' ' $a_{1}$, ' . . , ' $a_{n}$,' 'o,' ',', '=' are used to designate definite constituents of the system; on the other hand the capital letters, parentheses, and also words such as 'proposition' are to be taken as part of ordinary language. ${ }^{16}$ The capital letters, in

\footnotetext{
${ }^{15}$ On the omission of quotation marks here see Footnote 20.

${ }^{16}$ Ordinary language must here be understood as including the use of variables and parentheses in the way in which they are employed in mathematics.
} 
particular, are intuitive variables whose values are unspecified terms. Thus the specifications III $A$ are to be understood in the sense that for each particular determination of ' $A$,' ' $B$,' ' $C$,' each of the three propositions stated is an axiom; there are, therefore, infinitely many axioms subsumed under three axiom-schemes. ${ }^{17}$

Again, the conventions I, II, have a different character from III, and it is convenient to have a name for them collectively. I shall call these specifications and considerations based on them morphological, as opposed to the conventions III which will be called theoretical. The morphology of the system G has evidently a quite trivial character; in more sophisticated systems we may have a much more complicated morphology-involving different categories of terms, or even a whole hierarchy of types, relations between terms and definitions of complicated derived notions such as substitution. But we must suppose in all cases that the morphological specifications enable us to determine whether a given combination of symbols denotes a term (or an elementary proposition). ${ }^{18}$

These notions may be made somewhat clearer if I add a word or two concerning the interpretation. In the system $\mathrm{G}$ the $a_{1}, \cdots, a_{n}$ may be thought of as the generators of the group. On the other hand it may be shown that if in any theorem involving any $a_{i}$ we substitute

${ }^{17}$ This term is due to J. von Neumann, Zur Hilbertschen Beweistheorie, Mathematische Zeitschrift, vol. 26 (1927), pp. 1-46.

${ }^{18}$ It is to some extent arbitrary what considerations are taken as morphological; for in case of systems with a complicated morphology - say with different types of terms - we can take these types of terms as new predicates and transfer the rules concerning them into the theory. If this is carried to the limit we should arrive at a formal system, like the system G, in which there is only one category of terms, and it is not really necessary to state any morphological rules beyond giving the number of arguments for each operation and predicate. The natural formulation of the primitive frame on such a basis would be-

I. Primitive ideas.
A. Tokens (primitive terms).
B. Operations (classified as to number of arguments).
C. Predicates (do.).

II. Primitive theorems.
A. Axioms (that is, elementary primitive theorems).
B. Rules.

Such a system let us call a completely formal system. Evidently it is the more definite concept, and for a primary standard of mathematical rigor it forms the proper basis. $\mathrm{G}$ is an example of such a system. In most theories, however, there is a class of theorems, of a relatively trivial nature (from the point of view of that theory) which it is convenient to take unformalized. The concept of a formal system, with the possibility of some complication in the morphology, is thus more easily fitted to existing theories than that of a completely formal one. 
an arbitrary term $A$ for $a_{i}$, the result is again an elementary theorem. It follows that the $a_{i}$ may also be thought of as variables, and that the system contains those general equations of group theory which do not contain more than $n$ variables. To include all equations we must, naturally, postulate infinitely many primitive terms. If a substitution rule is added to the rules as Rule 6 , then the axiom schemes may be replaced by three single axioms as follows:

1. $a_{1} \circ\left(a_{2} \circ a_{3}\right)=\left(a_{1} \circ a_{2}\right) \circ a_{3}$,

2. $a_{1} \circ e=a_{1}$,

3. $a_{1} \circ a_{1}^{\prime}=e$.

The above formal system has of course a quite trivial character. (I do not claim that it is an adequate formalization of all of group theory.) On account of limitations of time, however, it will have to do as an example.

4. Discussion of the nature of a formal system. Let us now turn to the discussion of some points about the nature of a formal system. We shall inquire in what sense we may think of a formal system as an object, and whether we are justified in the use of the words 'proposition' and 'true' in connection with it. Also, on account of the prominence given to the question by Hilbert, it is necessary to say something about the relation of a formal system to symbolism; in this section and the next I shall defend the thesis that the extreme nominalism, with which formalism is now popularly associated, is no essential part of the doctrine.

As a preliminary to this discussion we must take account of the distinction between the use of a symbol as denoting something and the consideration of that symbol as an object. This is illustrated by the two sentences ${ }^{19}$

1. $\omega$ is an order type.

2. $\omega$ is a Greek letter.

From these it would be false to conclude that a Greek letter is an order type. Of course, under certain circumstances it is legitimate to use a symbol to designate itself. But, for maximum definiteness, I shall, when talking about a symbol (or expression), use a specimen of that symbol (or expression) enclosed in single quotation marks as a name for it. ${ }^{20}$

19 These are due to R. Carnap, Logical Syntax of Language, London and New York, 1937 (German edition, 1934), p. 156. Some changes in wording have been made.

20 This notation is due to Frege (see, e.g., Grundgesetze der Arithmetik, vol. 1, Jena, 1893, p. 4) and is now quite generally used by logicians. However, the distinction itself is made in some manner by linguists as a matter of course (and doubtless was before Frege's day). The distinction is important wherever the discussion has 
In setting up the primitive frame of a formal system we are, of course, introducing certain symbols; and, although we do not define these symbols in the ordinary sense, yet we do specify how they are to be used in connection with words of ordinary language (such as 'proposition' and 'true'). Thus, for G, in I A we say in effect that ' $e$,' ' $a_{1}$, ' . . , ' $a_{n}$ ' are nouns; we do not say what they are the names of, but they are names of some objects or other, which objects we call terms. Let us call these symbols the primitive term nouns. Then in I $\mathrm{C}$ we state that certain further expressions, formed from the primitive term nouns, the operators ' $O$ ' and ',' and parentheses, are also term nouns. II says that ' $=$ ' is a verb, and that elementary sentences are formed by placing it between two term-nouns. It is evident that

specific reference to the relation between symbols and their referents; but I think the confusion caused by non-observance of the distinction in ordinary mathematics is somewhat exaggerated. For discussion of this question cf. R. Carnap, Logical Syntax, pp. 156-160, or W. V. Quine, Mathematical Logic, New York, 1940, §4.

The discussion in the text has primary reference to symbols and expressions which are nouns. With respect to other types of expressions there should be at least a threefold, rather than a twofold, distinction. Thus, in connection with sentences we need to distinguish (a) the sentence as asserting something, (b) a noun naming the proposition asserted, and (c) a name for the sentence as a linguistic phenomenon. In the present paper I use the same expression in senses (a) and (b), relying upon the context to make the distinction clear. There is abundant precedent for this uage, both in ordinary and technical discourse, and it avoids overburdening the discussion with excessive symbolism. Nevertheless, a systematic method of making the distinction would be desirable. Such a method would be to use "corners," as in Quine's "quasi-quotation" (loc. cit., pp. 33-37, or Journal of Symbolic Logic, vol. 2, p. 146). This should be subject to the same rules as his quasi-quotation in regard to substitution for intuitive variables, so that, for example, we might write the conventions II of the primitive frame for $\mathrm{G}$ as-

There is one binary predicate, $r=.1$

If $A$ and $B$ are terms, then $\lceil A=B\rceil$ is an elementary proposition.

If this is coupled with an understanding of what symbols are intuitive variables we should have a satisfactory notation.

The distinction between (a) and (b) may be what the Principia Mathematica (and also Frege) had in mind in introducing the assertion sign ' $\vdash$.' However their explanations in this connection are somewhat vague. All that is clear is that ' $\vdash$ ' is placed before a noun to make a sentence-that is, it is an intransitive verb. It is therefore natural to use this symbol for the single unary predicate in formal systems which have such; it then corresponds to Hilbert's 'ist beweisbar.'

The neglect of the distinction between (b) and (c) seems to me to cause as much confusion as the neglect of that between (a) and (c). On this point compare the discussion of 'presentation' below.

It should be understood that single quotation marks, and these only, are used in the technical sense stated. Double quotation marks are used as in ordinary language. 
what has been said could be expanded into a way of stating the primitive frame, and that the new way of statement would be essentially equivalent to the preceding one.

The new form of statement, however-aside from the disadvantages inherent in its greater cumbersomeness - is a decided misplacement of the emphasis. To a mathematician the older form of statement indicates that the particular symbols used are irrelevant; whereas the newer form appears to be tied to a particular choice of them. Let us call a particular statement of the primitive frame, with a particular choice of symbols, a presentation of the system. Then the newer mode of statement connotes that we have to do with a particular presentation. On the other hand, a formal system is to be understood as an abstraction from its presentations. This does not mean that a formal system is a mystic entity subsisting apart from its presentations. Indeed the question of the philosophic nature of such an abstraction is irrelevant to the present discussion. All we need to know is that when we think of a presentation as that of a formal system we regard certain features as essential and others as irrelevant, and that what we have to say applies equally well to any other presentation agreeing in the essential features. A similar remark applies to the words 'proposition,' 'predicate,' and so on, as opposed to the corresponding linguistic words 'sentence,' 'verb' (or 'sentential function'), and so on. ${ }^{21}$

The next question is the indeterminacy which we have already noticed in regard to the terms. The primitive frame does not assign any definite objects to the term nouns. We are then free to make what determinations we please. Let us call any determination of such objects, which assigns a unique term-object to each term noun and conversely, a representation of the system. Then, given any presentation of the system, we can always supply a representation. In fact we can construct a mechanical model in which the tokens are different kinds of buttons and the operations are different ways of tying them together. A scheme of doing this for the system $G$ using auxiliary gadgets for the operations is shown by the diagrams. (Here it is to be understood that the holes are for the attachment of strings from the constituents $A, B$; while the rings are for attachment of a string connect-

${ }^{21}$ It is not even necessary that the essential features be precisely formulated. As ordinarily used 'proposition' is a vague word, in that no exact criteria are given for deciding when two sentences express the same proposition. This vagueness is harmless because we seldom or never have occasion to ask the question indicated. The word is useful because it indicates in a rough way the level of abstraction at which we are operating; which is better than no indication at all, or a misleading one. 
ing to an operation when the term $-A \circ B$ or $A^{\prime}$ is itself a constituent of a more complex term.)

When this is done the term nouns have been given a contensive meaning. Now the system does not admit of any further determination of such meaning. The indeterminacy which was noted for the terms does not extend to the predicates; for the rules II enumerate all of the

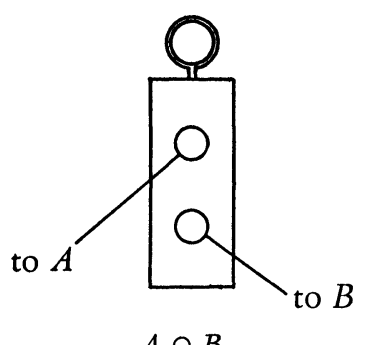

$A \circ B$

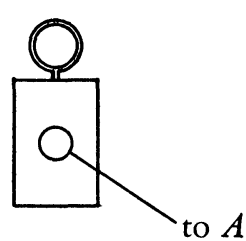

$A^{\prime}$

significant instances of these predicates and III all the true instances, so that the predicates are defined completely by the primitive frame. Thus in the above representation the pairs of artifacts between which the relation of equality holds are defined by the primitive frame and nothing else. Consequently although the terms are unspecified, yet for any formal system it is always possible to construct a representation supplying this deficiency. The elementary theorems hold without regard to how this is done. The representation is therefore an accident; and a formal system is an abstraction from its representations just as it is from its presentations.

The notion of representation just discussed must be distinguished from another notion, that of interpretation. We speak of an interpretation of a formal system when the elementary propositions are put into relation with certain contensive propositions independently defined. Thus we can interpret $G$ in a well known manner as a group of transformations, where two transformations are regarded as equal when they associate the same image with each point. This interpretation is a valid one, because the transformation processes associated with (formally) equal terms are also equal contensively; but the converse is not true. In the case of a representation such questions of validity cannot arise; all representations are isomorphic by definition.

A formal system, then, is something which is abstract in two ways; first, as to the symbols used to present it, and second, as to the objects used to represent it. The truth of the elementary propositions is in- 
dependent of these contingencies. Moreover by virtue of the conventions III (and the closure property mentioned at the beginning) an elementary proposition is true when and only when there exists a sequence of elementary propositions, of which the given proposition is the last, such that every proposition in the sequence is either an axiom or is derived from certain of its predecessors by a rule of deduction. What an elementary proposition asserts is, therefore, precisely the existence of such a proof. Assuming that the specifications for axioms and rules have a sufficiently definite character-which is to be taken as part of the definition of a formal system - the checking of an alleged proof is an objective process. ${ }^{22}$ An elementary proposition therefore states a question or problem such that an affirmative answer can be verified objectively, without any indeterminacy whatever.

5. The notion of a calculus. Although the notion of formal system is here taken as fundamental, yet other related notions are now more popular among specialists. As a typical such notion let us discuss here the notion of calculus as defined by Scholz. ${ }^{23}$

In a calculus it is explicitly stated that the objects we are dealing with are symbols. We start with a certain stock of symbols and with two kinds of rules for manipulating them. The first kind of rules, called formation rules, specifies recursively a certain set of expressions - that is, linear sets of symbols-which set I shall call formulas. The second set of rules, called transformation rules, specifies a class of formulas which I shall call assertible formulas; the rules consist of first a definite list of formulas, called here axiom formulas, which are assertible, and second, rules determining recursively how further assertible formulas are to be constructed.

Now it is evident that the rules of a calculus must be stated in a language such as English; and since the subject matter is symbols and expressions, the rules have somewhat the same character as the rules of syntax in grammar. For that reason the language is called the syntax language. On the other hand the expressions being talked about have also, in virtue of their being ordered by the rules, some of the properties of a language; and it is customary to refer to them as con-

\footnotetext{
${ }^{22}$ We may require of a proof that not only is the sequence of propositions stated, but the reasons for the inference at each stage.

${ }^{23} \mathrm{H}$. Scholz, Was ist ein Kalkill und was hat Frege für eine piinktliche Beantwortung dieser Frage geleistet, Semester Berichte (Münster), 7th Semester, Summer, 1935, pp. 16-22. The notion is similar to that of R. Carnap, Logical Syntax, pp. 4-8, $167 \mathrm{ff}$., but is somewhat more explicit.
} 
stituting the object language. There are thus two languages associated with a calculus, and the calculus may be thought of as a statement of the syntax of the object language in the syntax language. However the object language is a language only in a highly technical sense; it may or may not be a language in the usual sense.

Let us now consider the relations between a calculus and a formal system. We shall see that a formal system can be converted into a calculus, or something essentially similar, in two ways, either of which can be carried out mechanically. Conversely, by passing to a higher level of abstraction, a calculus can be converted into a formal system.

The process indicated in the discussion of presentation gives a method of forming a calculus from the symbols of the presentation. Thus, for the above presentation of the system $\mathrm{G}$, take as symbols ' $e$, ' $a_{1}$, ' . . . ' ' $a_{n}$,' 'о,' ',', '=,', '(,' ')' ; take as formulas the elementary sentences, and as assertible formulas those which express true propositions. Then, reinterpreting the rules, we have a calculus. This process I shall call calculization of the presentation. It is subject to the objections which were mentioned in the discussion of presentation.

Another way of reducing a formal system to a calculus is to represent the system symbolically. A uniform process for doing this is illustrated by the following procedure for $\mathrm{G}$; Let $e, a_{1}, \cdots, a_{n}$ be respectively ' $\epsilon$,' ' $\alpha_{1}$,' . . . ' ' $\alpha_{n}$,' and let ' $\phi$,' ' $\psi$,' be two new symbols; for given terms $A$ and $B$, let $A \circ B$ be the expression got by writing in order first ' $\phi$,' then $A$, then $B$; and let $A$ ' be obtained by prefixing ' $\psi$ ' to $A$. That this is a representation follows by certain results of Lukasiewicz. ${ }^{24}$ Then the terms will be a certain class of expressions which we can take as formulas. The resulting representation is not a calculus, because a relation between formulas and not a class of formulas is defined, but it has the essential nature of a calculus, and may be called a generalized calculus. ${ }^{25}$ The reduction would lead precisely to a calculus if the original system had a single unary predicate; and by a rather trivial change every formal system can be reduced to one of that character. ${ }^{26} \mathrm{By}$ this mode of reduction-as in any representation-the symbols of the primitive frame belong to the syntax

${ }^{24} \mathrm{~J}$. Lukasiewicz and A. Tarski, Untersuchungen über den Aussagenkalkul, Comptes Rendus de la Societé des Sciences et des Lettres de Varsovie, class III, vol. 23 (1930), p. 31. where references to earlier papers by Lukasiewicz (in Polish) may be found.

${ }^{25}$ The Church theory of conversion is an example of such a generalized calculus which occurs in the literature. See A. Church, Mathematical Logic, Princeton University (mimeographed), 1936, chap. 1.

${ }^{26}$ For the system $\mathrm{G}$ this can be done by introducing a new binary operation * and unary predicate $\vdash$, then replacing $A=B$ by $\vdash\left(A^{*} B\right)$. 
language, and the object language is the invented representation. ${ }^{27}$

In a calculus we do not consider all properties of the symbols, but only such as follow from the syntactical rules. Thus a calculus is to a certain extent abstract. We can make this abstractness explicit by converting to a formal system. In fact, using methods due to Tarski and Hermes ${ }^{28}$ we can find a (represented) formal system such that the tokens are the object symbols, the terms are the expressions, there is a single unary predicate, and the elementary theorems ascribe this predicate to those and only those expressions which are assertible formulas. We then have a formal system of a special kind with a symbolic representation. The object language can now be abolished altogether. Other processes of formalization - in which the terms are formulas or other special classes of expressions-may be possible; and if the calculus is obtained by calculization of a formal system, a process of formalization can be found which will bring us back to another presentation of the original formal system.

These considerations show that a formal system and a calculus are essentially equivalent notions. A calculus is, in fact, merely a formal system tied to a special representation. This representation is accidental. A formal system is just as exact a notion; it does not force emphasis on extraneous considerations; it requires only one set of symbols, and these are adjuncts to the language we use, not that which we talk about; it leaves the way open for representations in terms of some subject matter more suggestive than symbols; and a symbolic representation can be manufactured for it when needed. For these reasons the notion of formal system is here preferred as the fundamental notion of formalism.

So much for the notions of formal system and calculus. The important point is that for elementary propositions we have an objective criterion of rigor. We turn now to the consideration of propositions which are not elementary.

6. Metatheory. In the actual study of a formal system we do not confine ourselves to deducing elementary propositions step by step. Rather we take the formal system as datum, and, having defined the

${ }^{27}$ In the case of a completely formal system (see Footnote 16) with one unary predicate, this object language would consist of formulas and coordinating conjunctions only.

${ }^{28}$ E.g., see A. Tarski, Einige Betrachtungen über die Begriffe der w-Widerspruchsfreiheit und der $\omega$-Vollständigkeit, Monatshefte für Mathematik und Physik, vol. 40 (1930), pp. 97-112, and H. Hermes, Semiotik, Forschungen zur Logik und zur Grundlegung der exakten Wissenschaften, new series, vol. 5, 1938. 
object of study by setting up the primitive frame, we investigate it by any means available. In so doing we may formulate further propositions. I shall call these non-elementary propositions metapropositions (when true, metatheorems), and the method of study which gives rise to them the metatheoretic method.

We have already had an example of a metatheorem, namely, the substitution rule for the system $G$. It will be instructive to consider this theorem a little more carefully. First we need a precise definition of what we mean by the result of substituting the term $C$ for the token $a_{i}$ in the term $A$. Let us call this result $A^{*}$. Ordinarily we define it with reference to the symbols used to designate $A$ and $C$; but this apparent dependence on symbols can be avoided by defining $A^{*}$ recursively thus (here ' $\equiv$ ' denotes definitional identity):

1. $a_{i}^{*} \equiv C$;

2. If $A$ is a token distinct from $a_{i}, A^{*} \equiv A$;

3. $(A \circ B)^{*} \equiv A^{*} \circ B^{*}$;

4. $\left(A^{\prime}\right)^{*} \equiv\left(A^{*}\right)^{\prime}$.

The theorem, now, is the following: Suppose that $A$ and $B$ are terms such that

$$
A=B
$$

and $C$ is a term, then

$$
A^{*}=B^{*}
$$

Without going into details we may notice that the proof contains two essential steps. First, the conclusion is true if the hypothesis is an axiom; in fact then the conclusion is an axiom. Next, suppose the hypothesis is derived by a rule of procedure from certain premises, and that we have already shown that the theorem holds for these premises; then the same rule of procedure allows us to derive the conclusion from the transforms of the premises. At this point it is customary to say that the theorem follows by induction. However, what we have actually done is to exhibit a process whereby, given a proof of (1), we can convert it step by step into a proof of (2).

The variety of metatheorems is immense. Some further examples are the following. In the first place we may have simple combinations of the elementary propositions, namely,

$$
a_{1} \circ e=e \circ a_{1}=a_{1},
$$

which may be regarded as a conjunction of three elementary theorems. Then there are general theorems conjoining whole classes of 
elementary propositions such as

$$
A^{\prime} \circ A=e
$$

(the axiom schemes, considered as single propositions, are of this character); or derived rules, such as-

$$
\text { If } A \circ B=e \text {, then } B=A^{\prime} \text {. }
$$

Again we may introduce additional operations and predicates by recursive definitions, and consider general theorems concerning them, like the one just treated or the generalized associative law. Moreover there are properties of the system as a whole; such as consistency, completeness, and resolvability. We may also consider the relations of a system to other systems, in particular to its own subsystems and supersystems, its extensions of various kinds and so on. Here we tap the resources of modern algebra, with its extensions and ideals, including the four morph brothers-homo-, iso-, auto-, and holo-. Such metatheorems form the very life and soul of mathematics. Finally we may have considerations relating a system to extraneous (non-mathematical) considerations, idealistic hypotheses, or what not, such as the semantical investigations of Tarski and Carnap.

If the notion of formal system is taken as a basis, these metatheorems are expressed in the same language as the elementary theorems. Metatheory is thus not quite the same notion as Hilbert's metamathematics, although it is related to it. Hilbert sets up a calculus which may be regarded as the calculization of a portion of mathematics; and then calls the study of this calculus metamathematics. This is, to say the least, an unfortunate terminology. Hilbert, presumably, does not mean to imply that mathematics is merely a meaningless playing with the counters of his calculus, but rather that mathematics is an activity carried out in an interpreted object language from which meaning has been abstracted in the process of calculization; on the other hand his calculization does seem to imply, insofar as mathematics is formal, that the only legitimate mathematics is the explicit derivation of elementary theorems. But metatheorems have as good a right to be called mathematical as elementary ones; moreover from the general point of view which I shall mention later, Hilbert's metamathematics is a branch of mathematics rather than something which transcends it. Furthermore, we have already seen that if Hilbert's calculus is formalized we come back to another presentation of the original mathematics in the syntax language. The absolute separation of mathematics and metamathematics is fictitious. Hilbert's calculiza- 
tion had, I think, a psychological purpose, which it served well; but there is no sound basis for affirming the necessity of any such process.

As to the criteria of rigor for metatheoretic proofs, it is evident that we cannot handle the question as definitely as for the elementary ones. Many metatheoretic propositions have a vague character and require analysis. Such analysis can be given only by considering the specific kinds of metapropositions concerned. However, supposing this analysis made, we can divide metatheoretic proofs into two kinds, constructive and non-constructive. A constructive proof is one which, like the above proof of the substitution theorem, exhibits a process which can actually be carried through in any particular case arising under the hypotheses. For such a proof the criterion of rigor is reduced in the last analysis to that for the elementary propositions, and it has the same objective character. On the other hand the non-constructive proof depends on idealistic or other extraneous assumptions; and therefore we cannot regard them as purely formal.

An especially important class of metatheorems is constituted by certain theorems of incompleteness which have played a prominent role in recent years. These started with the Löwenheim ${ }^{29}$ theorem of 1915, which was later refined by Skolem. ${ }^{30}$ The upshot of these theorems was that any system of a certain kind would have an enumerable model in a sense which cannot be gone into here; since these systems have also interpretations which are non-enumerable, it follows that the interpretation cannot be unique as to structure. This, of course, is a non-constructive theorem. Sixteen years later Gödel ${ }^{31}$ proved constructively that in systems strong enough for the usual mathematical purposes there are elementary propositions such that, if the system is consistent, neither these propositions nor their formal negatives within the system can be proved. A similar theorem of Kleene and Rosser, published in $1935,{ }^{32}$ shows that a formal system cannot have

${ }^{29}$ L. Löwenheim, Über Möglichkeiten im Relativkalkul, Mathematische Annalen, vol. 76 (1915), pp. 447-470.

${ }^{30}$ Th. Skolem, Logisch-kombinatorische Untersuchungen über die Erfüllbarkeit oder Beweisbarkeit mathematischer Sätze nebst einem Theoreme über dichte Mengen, Videnskapsselskapet Skrifter, class I, 1920, no. 6.

${ }^{31} \mathrm{~K}$. Gödel, Über formal unentscheidbare Sätze der Principia Mathematica und verwandter Systeme, Monatshefte für Mathematik und Physik, vol. 38, pp. 173-198. For expositions of this famous theorem see also D. Hilbert and P. Bernays, Grundlagen der Mathematik, vol. 2, 1939, pp. 269-289, also J. B. Rosser, An informal exposition of proofs of Gödel's theorem and Church's theorem, Journal of Symbolic Logic, vol. 4 (1939), pp. 53-60.

${ }^{32}$ S. C. Kleene and J. B. Rosser, The inconsistency of certain formal logics, Annals of Mathematics, (2), vol. 36 (1935), pp. 630-636. A revised proof of this theorem is con- 
a kind of completeness as to term-formation and at the same time be capable of formalizing - in a sense which cannot be explained hereits own deductive processes.

7. Definition of mathematics. We are now in a position to discuss the formalist definition of mathematics. In this connection the incompleteness theorems just discussed have important consequences for the formalist conception of mathematics. Indeed they show that it is not feasible to consider mathematics as the development of a single formal system. There are other reasons, too, which support such a view; for the arbitrary nature of the definitions constituting the primitive frame of a formal system shows that, in principle at least, all formal systems stand on a par. ${ }^{33}$ The essence of mathematics lies, therefore, not in any particular kind of formal system, but in formal structure as such. Mathematics, then, should be defined as the science of formal systems in general; it should include all propositions elementary or metatheoretic, relating to one system or several, or to formal systems in general, so long as their criteria of truth depend on formal considerations alone.

This last proviso would exclude from mathematics proper all theorems depending on non-constructive proofs. This does not mean that these theorems are without mathematical interest. They are simply mixtures of mathematics and something else. As the example of the Löwenheim-Skolem theorem shows, these theorems are often of great significance for us; moreover they may lead to purely mathematical propositions at a higher level of formalization.

Mathematics, so conceived, has the following characteristics. First, it includes everything we know as mathematics. Classical analysis, for example, although not constructive when regarded as a metatheory of arithmetic, nevertheless can be embodied in a more inclusive formal system. ${ }^{34}$ Second, mathematics is a science, in that it consists of propositions - not formulas but real propositions, with a definite criterion of truth. This criterion of truth is capable of fully

tained in my paper The paradox of Kleene and Rosser (not yet published), and a considerable simplification in a paper, under the same title as that of Kleene and Rosser, which is now in preparation.

${ }^{33}$ This statement is not to be confused with the naive view that mathematics consists of more or less autonomous postulate systems. For if we could formulate a completely adequate logic, then these postulates could be added to the hypotheses of the theorems, and so all the postulate systems could be subsumed under one mastersystem-logic. But the point is that we can not have any such logic.

${ }^{34}$ Such formal systems, insofar as they are known at present, are not wholly satisfactory. Cf. Church's appendix to F. Gonseth, Philosophie Mathématique, Paris, 1940. 
objective verification or proof-the primary standard of rigor for such a proof being the definition of derivability of an elementary proposition. On the other hand the gist of mathematics is that we make definitions by recursion, and then draw particular consequences by applying the definitions and general consequences by mathematical induction. There is thus a certain amount of justice in the view that mathematical theorems are consequences of definitions; but since the definitions are recursive, mathematics does not have the trivial character which that seems to imply. Likewise there is justice in the view that mathematical induction is a process of cardinal importance. However, it is not a mysterious power of the human mind, as Poincaré seemed to think, but is simply a corollary of recursive definitions; for if we demonstrate by mathematical induction that all members of a recursively defined class have a certain property, that demonstration is constructively valid because by definition every member of the class can be reached by the inductive process.

At an earlier stage I stated that most of the positive criticisms of the intuitionists find their justification in modern formalism. We have seen that this is so for the Kronecker demand for constructivity; indeed we are more stringent in this particular than the intuitionists themselves. ${ }^{35}$ In the preceding paragraph we have also seen that their emphasis on mathematical induction has some justification. As for the law of excluded middle, it is necessary first to explain what it means. If we interpret it as a metaproposition concerning a formal system $S$, to the effect that every elementary proposition of $S$ is true or false, then we need to know the meaning of 'false' (not to mention 'or'). Now although we know what it means to verify an elementary proposition of $S$, yet we have no constructive definition of what it means to say it is false. We might define constructive falsity in any one of a number of different ways; but for any of the suggested definitions the law of excluded middle holds only if the system has a relatively trivial character ${ }^{36}$

There is not time to go further with this. We can say, however, that it is useless to deny that intuition is involved in mathematicsif one defines intuition properly the statement is a tautology. However, we do not have to postulate a metaphysical character for it;

${ }^{35}$ This is shown by Gödel's reduction of classical arithmetic to that of intuitionism (Zur intuitionistischen Arithmetik und Zahlentheorie, Ergebnisse eines mathematischen Kolloquiums, vol. 4 (1932), pp. 34-38). The consistency of classical arithmetic cannot be proved constructively in any such simple manner.

${ }_{36}$ These matters I plan to discuss more fully in a forthcoming paper Some properties of formal deducibility. 
indeed to many of us it seems that this intuition is an empirical, linguistic phenomenon. On the other hand if we subtract from intuitionism its metaphysics, the remaining differences between it and formalism are relatively superficial as regards mathematical rigor. The main point is that the intuitionist prefers to confine attention to certain special types of formal systems. This leads to considerations, going beyond the definition of rigor, to which we must now devote some attention.

8. Acceptability. It is obvious that we are not interested in all formal systems. Those considerations which lead us to choose one system rather than another need to be distinguished from those relating to the truth of propositions concerning a formal system once chosen. To make this distinction I shall call that property of a formal system which leads us to adopt it for consideration its acceptability. This is evidently a quasi-truth concept which applies to a system as a whole.

In strictness acceptability is irrelevant to the problem of mathematical rigor. A proof of a proposition relating to a formal system is rigorous if and only if it satisfies certain objective criteria which are independent of acceptability. But it is necessary to give a brief account of acceptability for two reasons: first, because there has been some confusion in regard to it, and second because it sheds some light on the significance of mathematical rigor from the point of view of science.

The first point is that acceptability is relative to a purpose. It usually means that we are interested in some interpretation of the formal system; and the validity of the interpretation in relation to the subject matter is the prime consideration. Without statement of the purpose any discussion of acceptability is futile. Moreover the various schools of thought are not necessarily in conflict with one another. There is no one absolutely acceptable system; for different purposes entirely different systems may be acceptable.

Again if the purpose is an empirical one, as it is in physics, the question of acceptability is empirical also. Neither intuitive evidence, which is stressed by the intuitionists, nor demonstrable consistency, which is insisted on by Hilbert, is of more than secondary importance. From this point of view I think we must agree that, at the present state of our knowledge, some system of classical analysis is acceptable, however meaningless it may be to the intuitionists, and however far we may be from a formulation with a consistency proof. Of course if an inconsistency should be found, we should have to modify the system; but at the present time the acceptability of analysis is an empirical fact which requires no justification. 
It is perhaps worth mentioning that the demand for intuitive evidence and the demand for consistency proof amount, in the last analysis, to nearly the same thing. For a thoroughly satisfactory consistency proof would exhibit a process whereby, given a classical proof with an intuitionistically significant conclusion, we could transform it into an intuitionistic proof of the same conclusion. Ackermann's recent proof of the consistency of arithmetic ${ }^{37}$ does just this for a certain class of propositions. The proof is accomplished by a series of total replacements ("Gesamtersetzungen"); if the conclusion of the original proof is a particular numerical formula, the last total replacement gives an intuitive proof of the same formula. A complete solution of the consistency problem for analysis would show that any classical proof of a proposition with a direct intuitive meaning could be transformed into a purely constructive proof. In view of the Löwenheim-Skolem theorem there is reasonable doubt as to the possibility of such a transformation. And even if we succeed in finding it, it is not improbable that we shall always have uses for systems whose consistency is unknown.

The upshot of this is that we should cultivate a tolerant attitude in matters of acceptability. ${ }^{38}$ Acceptability is not a question of right and wrong but of choice of subject matter. Such a choice should be free; and some difference of opinion is not only allowable but desirable. As mathematicians we should know to what sorts of system our theorems - if formalized-belong; but to exclude systems which fail to satisfy this or that criterion of acceptability is pedantry.

From the point of view of formalism, then, the question of truth of a naive mathematical proposition can be split into two parts. The first is the discovery of the formal system to which the proposition is to be referred, and the proof of the corresponding formal proposition; this is the problem of mathematical rigor, and we have seen that it has an objective character. The second is the acceptability of that system for the purpose in hand; this is a problem of applied mathematics.

Pennsylvania State College

${ }^{37} \mathrm{~W}$. Ackermann, Zur Widerspruchsfreiheit der Zahlentheorie, Mathematische Annalen, vol. 117 (1940), pp. 162-194.

${ }^{38}$ Cf. Carnap's "Principle of Tolerance" (Logical Syntax, p. $51 \mathrm{ff}$.); also Weyl, H., Die heutige Erkenntnislage in der Mathematik, Sonderdrucke des Symposion, vol. 3, Erlangen, 1926, pp. $31 \mathrm{ff}$. 